\title{
Non-Exponential Decays in First-Order Kinetic Processes. The Case of "Squeezed Exponential"
}

\author{
J. Sworakowski And K. MATCZYSZYN \\ Institute of Physical and Theoretical Chemistry \\ Wrocław University of Technology \\ Wybrzeże Wyspiańskiego 27, 50-370 Wrocław, Poland \\ Dedicated to the memory of late Professor Jerzy Prochorow
}

\begin{abstract}
Kinetics of processes, in which the reaction rate increases with conversion, is discussed and illustrated with an example of the chemical reaction of isomerization of an azobenzene derivative in a liquid crystalline matrix. A simple phenomenological model is put forward explaining the effect by dynamic changes of interactions between the reacting species and the matrix.
\end{abstract}

PACS numbers: 82.20.Pm, 82.30.Qt

\section{Introduction}

Kinetics of many chemical and physical processes is well described by the first-order equations, according to which the conversion rate is proportional to the number (concentration) of unreacted species

$$
\frac{\mathrm{d} x}{\mathrm{~d} t}=k(1-x)
$$

where $k$ is the rate constant, and $x$ is the conversion ratio, i.e., the ratio of the number (concentration) of reacted species to the initial number (concentration) of reactant molecules. In thermally activated processes, $k$ is temperature dependent, following the Arrhenius equation

$$
k=\nu \exp \left(-\frac{E}{k_{\mathrm{B}} T}\right),
$$

with $k_{\mathrm{B}}$ standing for the Boltzmann constant, $\nu$ - for the frequency factor, and $E$ being the activation energy. The latter two parameters (and hence the rate constant) are usually assumed conversion-independent. Under this assumption, the solution of Eq. (1) reads 


$$
1-x=\exp (-k t)
$$

Thus the plot of $\ln (1-x)$ vs. $t$ should yield a straight line, its slope amounting to $-k$ (cf. Fig. 1). This is a common "textbook approach" to the first-order kinetics (cf., e.g. [1, 2]). Quite often, however, processes expected to follow the first-order kinetics, do not obey Eq. (3): in most cases, the rate of the process decreases as the conversion increases. The decays in this case can often be described by the phenomenological "stretched exponential" (Kohlrausch-Williams-Watts, KWW) equation $[3,4]$

$$
1-x=\exp \left(-(\kappa t)^{\alpha}\right),
$$

with $\kappa$ standing for the effective rate constant and $\alpha$ being a time-independent parameter $(0<\alpha<1)$. Several explanations have been put forward to explain the latter behaviour (see, e.g. [5-10] and references therein). In the case of processes taking place in constrained media (solutions of reacting species in viscous liquids, matrices, etc.), a commonly accepted model assumes a distribution of rate constants resulting from a distribution of microenvironments of reacting species: a spread of intermolecular distances and orientations modifies the frequency factor and/or activation energy. It is important to point out that the distribution has been assumed static, i.e., conversion-independent. In other words, an elementary reaction event taking place on a molecule is not supposed to affect other unreacted molecules. One may demonstrate that such a static distribution always yields decays that can be approximated by the stretched exponential with $\alpha<1$, the parameter $\alpha$ depending on the distribution width.

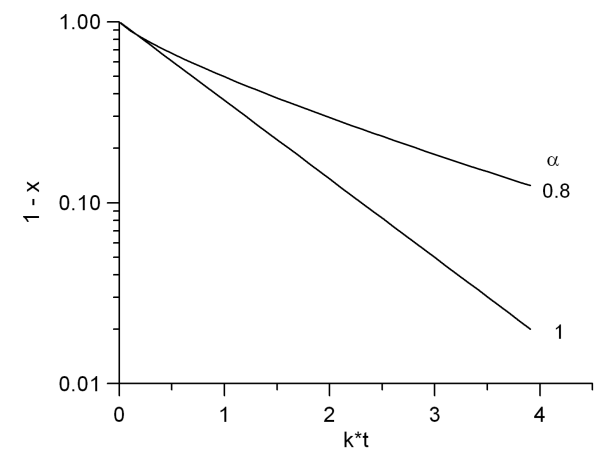

Fig. 1. Calculated decay curves: an exponential decay (Eq. (3) or Eq. (4) with $\alpha=1$ ), and a stretched exponential decay with $\alpha=0.8$.

There exist, however, processes whose kinetics is evidently of the first order in "normal" environments but which exhibit an unconventional behaviour in, e.g., liquid crystalline matrices. Isomerization of azobenzene derivatives may serve as a good example. 


\section{Experimental example}

The azobenzene family is one of the best known photochromic systems (cf., e.g. $[11,12]$ and references therein), and kinetic parameters of the isomerization have been determined by several researchers (e.g. [13, 14]). The thermally driven cis-trans reaction follows the first-order kinetics in common solvents [15, 16], whereas one can observe a stretched exponential behaviour in diluted solutions in polymer matrices [17-19]. We have found, however, that the kinetics of the same reaction in liquid crystalline matrices is, in some cases, qualitatively different and cannot be described by the equations mentioned above.

The experiment reported in this note is a thermally driven cis-trans isomerization of 4-fluoro-4'-methoxyazobenzene (hereafter refered to as FMA) in liquid-crystalline 4-hexylo-4'-cyanobiphenyl (6CB) (cf. Fig. 2a). The reaction changes the spectrum of the system (see Fig. $2 \mathrm{~b}$ ), hence one may monitor the progress of the isomerization.

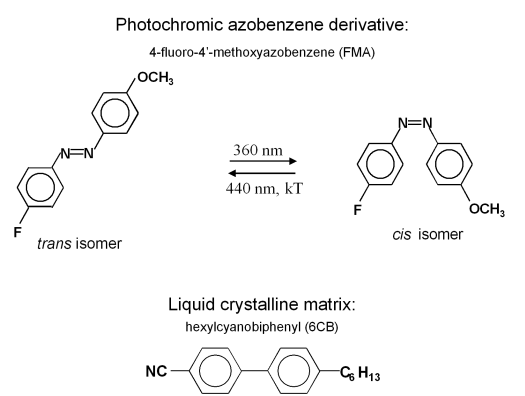

(a)

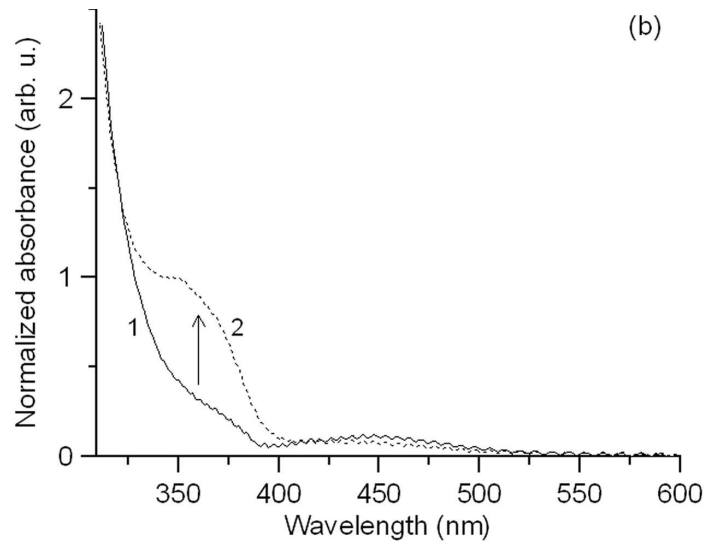

(b)

Fig. 2. (a) Chemical formulae of the system under study (FMA in 6CB), and a scheme of the isomerization of FMA. (b) Absorption spectra of the system under study in the visible spectral range. Curve 1: the solution immediately after UV irradiation (most of FMA converted into the cis form); curve 2: the solution in thermal equilibrium (FMA converted into the trans form). The sharp increase in absorbance below $350 \mathrm{~nm}$ is due to the absorption of $6 \mathrm{CB}$. The curves were normalized to the peak absorbance at $t=\infty$.

The temporal evolution of the absorbance of FMA, measured at $360 \mathrm{~nm}$ (i.e., near the maximum of the absorption of the trans form) is shown in Fig. 3a.

The curve shown in the figure can be simply converted into the temporal change of the concentration of cis-FMA by making use of the Lambert-Beer law: it is straightforward to show that the following relation holds:

$$
1-x=\frac{A(\infty)-A(t)}{A(\infty)-A(0)}
$$

where $A(0), A(t)$, and $A(\infty)$ are initial, momentary, and final absorbances, respec- 

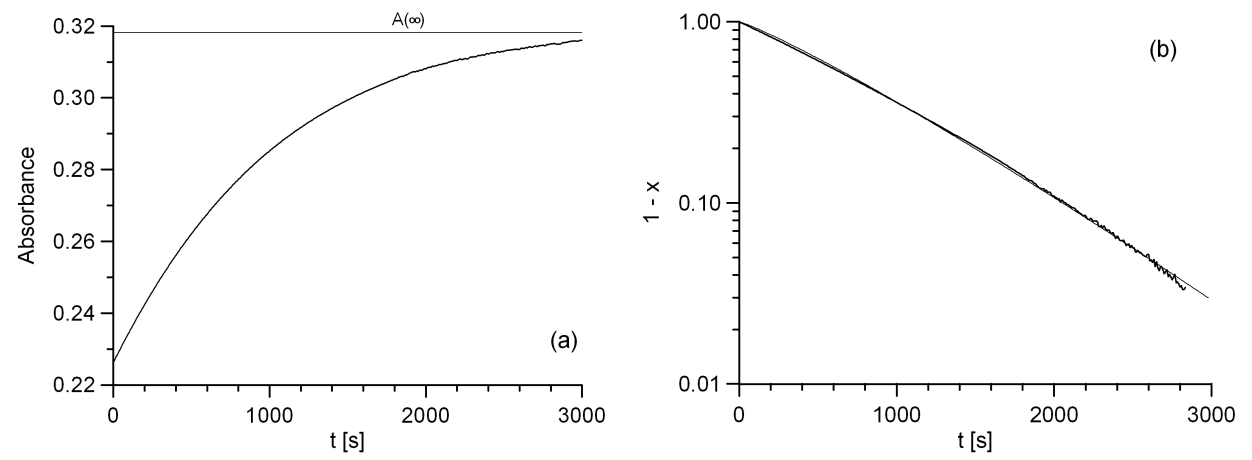

Fig. 3. (a) Temporal evolution of the absorbance of the solution of FMA in 6CB ( $T=$ $301 \mathrm{~K}, c=10^{-5} \mathrm{~mol} \mathrm{dm}{ }^{-3}$ ). (b) Solid line: the data re-calculated using Eq. (5) and plotted in semilogarithmic coordinates; dotted line: the data fitted with Eq. (4) with $\alpha=1.13$.

tively. The resulting curve, shown in Fig. 3b, is clearly non-exponential but the increasing slope of the curve shows that, contrary to the case of stretched exponential, the rate of the reaction increases with progressing conversion; by analogy to "stretched exponential", such a behaviour may be referred to as "squeezed exponential". The dependence shown in Fig. 3b can be fitted with Eq. (4), the fit yielding $\alpha=1.13>1$.

\section{The model}

The behaviour exemplified with the curve shown in Fig. 3b cannot be explained within the model assuming static distributions of the parameters determining the rate constant. Thus one should seek another explanation, the most obvious one being conversion-depending rate constant. In other words, one should assume that the reaction changes microenvironment of unreacted molecules, hence dynamically modifying the activation energy and/or frequency factor as the reaction progresses.

In the following, we shall adopt a simple phenomenological model assuming a linear dependence of $E$ and $\ln \nu$ on conversion

$$
E=E_{0}+\varepsilon x, \quad \ln \nu=\ln \nu_{0}+\beta x,
$$

where $\varepsilon$ and $\beta$ are conversion-independent parameters. We will neglect a possible effect of distributions of the rate constants.

Equation (2) thus becomes

$$
k=\nu_{0} \exp \left(-\frac{E_{0}}{k_{\mathrm{B}} T}\right) \exp (\gamma x)=k_{0} \exp (\gamma x),
$$

where $\gamma=\beta-\varepsilon / k_{\mathrm{B}} T$.

Since $k$ is now conversion-dependent, Eq. (1) can be, in general, solved only numerically. We shall, however, limit ourselves to the case of $|\gamma|<1$; Eq. (7) can then be approximated by 


$$
k \approx k_{0}(1+\gamma x) .
$$

Equation (1) can now be rewritten in the form

$$
\frac{\mathrm{d} x}{\mathrm{~d} t}=k_{0}(1-x)(1+\gamma x) .
$$

The solution of Eq. (1a) reads

$$
1-x=\frac{(1+\gamma) \exp \left(-k_{\mathrm{eff}} t\right)}{1+\gamma \exp \left(-k_{\mathrm{eff}} t\right)}
$$

where $k_{\text {eff }}=k_{0}(1+\gamma)$. Let us note that the above equation becomes identical with Eq. (3) for $\gamma=0$.

Plots of $\ln (1-x)$ vs. $t$ are shown in Fig. 4a. As is clearly seen in the figure, for positive values of $\gamma$, the curves apparently follow the "squeezed exponential" kinetics. On the other hand, for $\gamma<0$ the curves resemble stretched exponential ones.
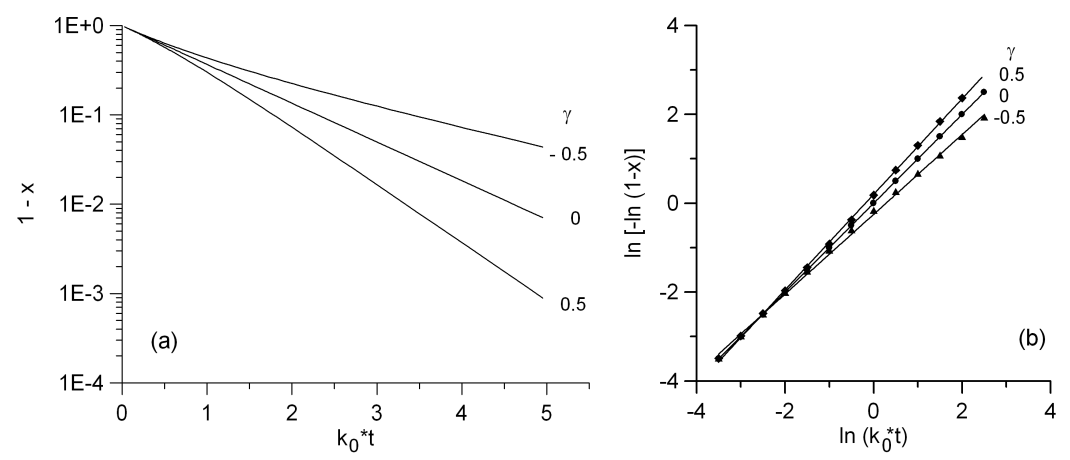

Fig. 4. (a) Decays calculated using Eq. (8) with $\gamma$ equal to -0.5 (stretched exponential), 0 (exponential) and +0.5 (squeezed exponential); (b) the curves of (a) re-plotted in the coordinates linearizing Eq. (4); the slopes are equal to the values of $\alpha$. Triangles: $\gamma=-0.5$; circles: $\gamma=0$; diamonds: $\gamma=0.5$ (only every 10th point is shown). The lines are fits to the points with Eq. (4).

The curves shown in Fig. 4a have been re-plotted in the coordinates proving that they can indeed be fitted with Eq. (4) for both, negative and positive values of $\gamma$, over a wide range of times and conversions (Fig. 4b). The resulting relation between $\gamma$ and the parameter $\alpha$ is shown in Fig. 5.

\section{Discussion and final remarks}

The results presented in the preceding section point to factors behind the unusual kinetic behaviour of certain systems: the primordial factor seems to be a dynamic change of the rate constant occurring during the reaction, associated with changes in the microenvironment of reacting molecules. Both, "stretched exponential" and "squeezed exponential" kinetics can be obtained, depending on 


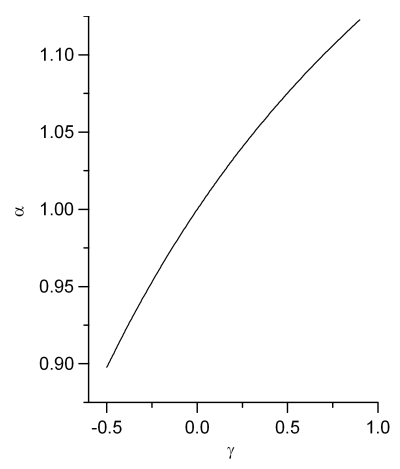

Fig. 5. Relation between the parameter defined in Eq. (7) and the parameter $\alpha$ defined in Eq. (4). Let us note the "squeezed exponential" behaviour $(\alpha>1)$ expected for positive values of $\gamma$.

whether the rate constant is a decreasing or increasing function of the reaction progress. Whilst the former behaviour can also be due to static distributions of frequency factors and/or activation energies, the latter kinetics must only result from the dynamic changes.

The simple model put forward in this note does not aim at seeking a quantitative agreement with experimental data. The results provided raise, however, a fundamental question about the microscopic mechanism of the "squeezed exponential" kinetics. The model implicitly assumes that the act of conversion of an individual molecule affects the environment of other (unreacted) ones. At the concentration level of the order of $1 \%$ or less, this means that the result of the conversion act is felt at distances of the order of several nm. One may speculate that a cooperative process occurring in the liquid crystalline matrix is involved, similar to (or even identical with) that found by the authors of this contribution $[20,21]$ and by other researchers [22] in dye doped liquid crystals. It has been found that the reaction of a photochromic solute affects the phase stability of the liquid crystalline matrix. This means that it should affect the long-distance ordering of the matrix to a degree depending on the progress of the reaction, and hence may dynamically modify the kinetic parameters of the reaction. The concept presented here, however, is only a speculation, and a further research is necessary to put forward a more advanced model.

\section{Acknowledgments}

The research was supported by the Wrocław University of Technology.

\section{References}

[1] P.W. Atkins, Physical Chemistry, 6th ed., Oxford Univ. Press, Oxford 1998, Ch. 25.

[2] K. Pigon, Z. Ruziewicz, Physical Chemistry, 5th ed., PWN, Warsaw 2005, Vol. 1, Ch. 7 (in Polish). 
[3] R. Kohlrausch, Ann. Phys. (Leipzig) 12, 393 (1947) (cited after [4]).

[4] G. Williams, G.D. Watts, Trans. Faraday Soc. 66, 3348 (1970).

[5] A. Płonka, Ann. Rep. Prog. Chem. C, Phys. Chem. 91, 107 (1994).

[6] A.K. Jonscher, Universal Relaxation Law, Chelsea Diel. Press, London 1996.

[7] Special Issue Rate Processes with Kinetic Parameters Distributed over Time and Space, Eds. Yu.A. Berlin, J.R. Miller, A. Płonka, Chem. Phys. 212, 1 (1996).

[8] J.C. Phillips, Rep. Prog. Phys. 59, 1133 (1996).

[9] A. Płonka, Ann. Rep. Prog. Chem. C, Phys. Chem. 94, 89 (1997).

[10] J. Sworakowski, K. Janus, S. Nešpürek, Adv. Colloid Interface Sci. 116, 97 (2005).

[11] H. Rau, in: Photochromism. Molecules and Systems, Eds. H. Dürr, H. Bouas-Laurent, Elsevier, Amsterdam 1990, Ch. 4.

[12] Special Issue Azobenzene-Containing Materials, Ed. A. Natansohn, Macromol. Symp. 137, 1 (1999).

[13] G.S. Hartley, J. Chem. Soc., 633 (1938).

[14] E.R. Talaty, J.C. Fargo, Chem. Commun., 65 (1967).

[15] G.M. Wyman, Chem. Rev. 54, 625 (1955).

[16] G. Zimmerman, L.-Y. Chow, U.-J. Paik, J. Am. Chem. Soc. 80, 3528 (1958).

[17] K. Janus, J. Sworakowski, E. Luboch, Chem. Phys. 285, 47 (2002).

[18] K. Janus, I.A. Koshets, J. Sworakowski, S. Nešpürek, J. Mater. Chem. 12, 1657 (2002)

[19] K. Janus, J. Sworakowski, J. Chem. Phys. B 116, 302 (2005).

[20] K. Matczyszyn, J. Sworakowski, J. Chem. Phys. B 107, 6039 (2003).

[21] K. Matczyszyn, A. Chwiałkowska, J. Sworakowski, Thin Solid Films, in press.

[22] T. Sasaki, T. Ikeda, K. Ichimura, Macromolecules 26, 151 (1993). 